\title{
Correlations in a one-dimensional Bose gas
}

\author{
E. Demirel and B. Tanatar \\ Department of Physics, Bilkent University, Bilkent, 06533 Ankara, Turkey
}

(Received 11 June 1998)

\begin{abstract}
We study the correlation effects in a one-dimensional Bose gas with repulsive delta-function interaction. The correlation effects are described by a local-field correction which takes into account the short-range correlations. We find that the ground state energy is in good agreement with the exact result up to intermediate coupling strengths, showing an improvement over the Bogoliubov and other related approximations. The velocity of sound, the static structure factor, and pair-correlation function are also calculated within the present approximation. [S0163-1829(99)00314-8]
\end{abstract}

\section{INTRODUCTION}

The one-dimensional (1D) electron gas models are attracting a lot of interest because of theoretical and technological implications, since the motion of electrons confined to move freely only in one spatial dimension gives rise to a variety of novel phenomena, such as the non-Fermi liquid characteristics. ${ }^{1}$ The system of bosons are of equal importance because of the role played by statistics and the revelation of macroscopic effects like Bose-Einstein condensation and superfluidity. ${ }^{2}$ The interplay between the statistics and interaction effects, the enhanced quantum fluctuations in low-dimensional systems, and the prospects of experimental realization provide ample motivation to study quantum many-body systems in $1 \mathrm{D}$.

A system of bosons in one-dimension interacting via a short-range, delta-function potential has been a useful model to study the nature of ground state properties of quantum systems and assessing the role played by statistics in comparison to the corresponding system of 1D fermions. ${ }^{3,4}$ The close analogy between the fermions and bosons in 1D has been established. ${ }^{4}$ An exact analysis of the ground-state properties, in particular the ground-state energy as a function of the coupling strength, of a 1D interacting Bose gas was provided by Lieb and Liniger. ${ }^{5,6}$ Yang and Yang $^{7}$ extended this Bethe ansatz approach to study the thermodynamics at finite temperature. Correlation effects in a 1D Bose gas within the self-consistent field approximation was first attempted by Hipôlito and Lobo ${ }^{8}$ and recently by Gold. ${ }^{9}$ The many-body effects beyond the mean-field theory was described by the local-field correction calculated within the Singwi et al. ${ }^{10}$ (STLS) scheme. The local-field corrected Bogoliubov approximation ${ }^{11}$ shows a definite improvement for the ground state energy. Recently, Kerman and Tommasini ${ }^{12}$ introduced a Gaussian time-dependent variational principle for bosonic systems and applied their method to the problem of $1 \mathrm{D}$ bosons interacting through a repulsive contact potential. Charged bosons in $1 \mathrm{D}$ have also attracted some interest. ${ }^{13}$ Correlation effects in a quasi-one-dimensional charged Bose condensate is also studied within the STLS scheme. ${ }^{14}$ There has been a renewed interest in low density ${ }^{15}$ Bose gases because of the recent experimental progress in achieving Bose-Einstein condensation in atomic vapors under external potentials. ${ }^{16}$ It is expected that by adjusting the confining potential in these atomic systems, two and possibly one-dimensional boson condensates may be produced.

In this work we revisit the problem of $1 \mathrm{D}$ bosons interacting via a delta-function potential within the local-field correction approach. We describe the correlation effects in the interacting Bose gas in terms of a local-field factor introduced by Vashishta and Singwi ${ }^{17}$ (VS) in an approximate way. We find that the exact ground-state energy ${ }^{5}$ within this perturbation theory approach can be faithfully reproduced up to large values of the coupling constant. This is an improvement over the Bogoliubov approximation ${ }^{11}$ and the STLS scheme $^{10}$ which show agreement with the exact ground state energy for small values of the coupling strength. Although based on certain approximations, the perturbation theory approaches have the advantage of obtaining the ground-state properties, and in particular the correlation functions in the weak and intermediate coupling regimes. These may also find their use in other applications. The self-consistent approximation scheme of Singwi et al. ${ }^{10}$ makes use of the oneparticle distribution function in deriving the density response of the many-body system to an external perturbation. Since the equation of motion of the one-particle distribution function depends on the two-particle distribution function, and so on, the hierarchy of equations is truncated by making an assumption for the two-particle distribution function. The approach developed by Vashista and Singwi ${ }^{17}$ is also based on a similar idea but introduces a slightly different assumption at the truncation level. More explicitly, the instantaneous pair-correlation function is approximated as the equilibrium pair-correlation function and a term involving its derivative with respect to the density which amounts to a first order correction in the density fluctuation $\delta n$. The VS approach has recently been applied to a degenerate plasma of charged bosons. ${ }^{18}$

An important limitation of the present approach which is shared by the Bogoliubov and STLS approximations is that the system is assumed to be in the Bose-Einstein condensed state. Since it is established ${ }^{19}$ that the homogeneous one- and two-dimensional systems cannot have long-range order (hence no condensate) the above assumption violates the correct behavior of interacting systems in 1D. As we show in the sequel, the ground-state energy and some correlation functions are nevertheless determined quite reliably. The method is not appropriate to account for the long-range cor- 
relations responsible for the behavior of the momentum distribution, whereas the other correlation functions such as the static structure factor and pair-correlation function are expected to be less severely influenced by this assumption.

The rest of this paper is organized as follows. In the next section we present the model of 1D bosons and the theoretical framework with which to discuss the correlation effects. In Sec. III we calculate the ground state energy of the system and compare with the exact result and other related approaches. Sections IV and V discuss the collective excitations and the compressibility sum-rule, respectively. The correlation functions within the present model are calculated in Sec. VI. We conclude in Sec. VII with a brief discussion of our results.

\section{MODEL AND THEORY}

We consider a system of bosons in 1D interacting via a contact potential $V\left(r_{1}, r_{2}\right)=V_{0} \delta\left(r_{1}-r_{2}\right)$, where $V_{0}$ is the interaction strength. In terms of the boson mass $m$ and the density of the particles $n$, we use the dimensionless parameter $\gamma=m V_{0} / n$ to characterize the strength of the coupling (we take $\hbar=1$ ). After Hipôlito and Lobo $^{8}$ and Gold $^{9}$ we further assume that the 1D bosons are in the condensate and the generalized Bogoliubov model is applicable. The localfield concept has been demonstrated by Gold ${ }^{9}$ to be quite useful in understanding the weak coupling regime of 1D bosons. The ground state properties were calculated to be in good agreement with the exact results of Lieb and Liniger ${ }^{5,6}$ as $\gamma \rightarrow 0$.

In this work we choose a different local-field factor in the description of the ground state correlation effects for 1D bosons. We use the approach introduced by Vashishta and Singwi ${ }^{17}$ (VS) which was originally constructed to satisfy the compressibility sum-rule. As discussed in Sec. I, in the modified theory of Vashishta and Singwi ${ }^{17}$ the equilibrium pair correlation function $g(r)$ which enters the ansatz for the two-particle distribution function is amended by a correction term involving the density derivative of $g(r)$. For a onedimensional system of bosons interacting via a constant potential (in the wave vector space) the local-field factor in the Vashishta-Singwi approximation now reads

$$
G_{\mathrm{VS}}(\gamma)=\left(1+a n \frac{\partial}{\partial n}\right) \frac{1}{n \pi} \int_{0}^{\infty} d q[1-S(q)]
$$

where $a$ is an adjustable parameter. Note that the local-field factor $G$ is still independent of the wave vector variable as in the STLS approximation. As in the case of the STLS scheme, the above equation for $G$ has to be solved selfconsistently along with the static structure factor $S(q)$ given in the generalized Bogoliubov approximation by ${ }^{9}$

$$
S(q)=\left[1+\frac{4 n^{2}}{q^{2}} \gamma(1-G(\gamma))\right]^{-1 / 2}
$$

Substituting Eq. (2) into Eq. (1), we obtain the following differential equation for $G_{\mathrm{VS}}(\gamma)$

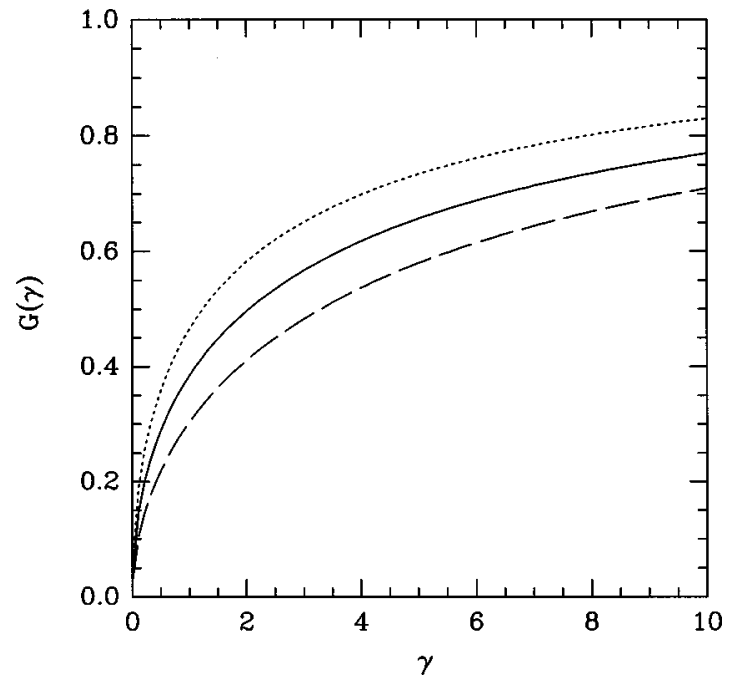

FIG. 1. The local-field factor $G_{\mathrm{VS}}(\gamma)$ at $a=1 / 2$ (solid line) and $a=1$ (dashed line), and $G_{\mathrm{STLS}}(\gamma)$ (dotted line) (Ref. 9) as a function of $\gamma$.

$$
\frac{d G_{\mathrm{VS}}}{d \gamma}=\frac{\pi}{a \gamma^{3 / 2}} G_{\mathrm{VS}}\left(1-G_{\mathrm{VS}}\right)^{1 / 2}+\frac{(1-2 / a)}{\gamma}\left(1-G_{\mathrm{VS}}\right),
$$

which is first order, but highly nonlinear. Rather than attempting to solve Eq. (3) numerically, we adopt a simpler approximation given by

$$
G_{\mathrm{VS}}(\gamma)=\left(1-a \gamma \frac{\partial}{\partial \gamma}\right) G_{\mathrm{STLS}}(\gamma),
$$

which is the lowest-order expression in the iterative solution of Eqs. (1) and (2), starting from the STLS solution. As we shall see later, the lowest-order approximation is capable of improving the STLS approach to the ground-state energy remarkably, keeping the discussion at the same level of transparency given by Gold. ${ }^{9}$ The closed form expressions for $G_{\text {STLS }}$ (Refs. 8 and 9) and $G_{\mathrm{VS}}$ (within our approximate scheme) are easily obtained to be

$$
G_{\mathrm{STLS}}(\gamma)=\frac{2 \gamma}{\pi^{2}}\left[\left(1+\pi^{2} / \gamma\right)^{1 / 2}-1\right]
$$

and

$G_{\mathrm{VS}}(\gamma)=\frac{2}{\pi^{2}}(1-a) \gamma\left[\left(1+\pi^{2} / \gamma\right)^{1 / 2}-1\right]+a\left(1+\pi^{2} / \gamma\right)^{-1 / 2}$

respectively. In Fig. 1 we display the local field correction $G_{\mathrm{VS}}(\gamma)$ for $a=1 / 2$ and $a=1$, and $G_{\mathrm{STLS}}(\gamma)$ as a function of the coupling strength $\gamma$. The weak coupling limit of $G_{\mathrm{VS}}(\gamma)$ is $G_{\mathrm{VS}}(\gamma \rightarrow 0) \approx(2 / \pi) \gamma^{1 / 2}(1-a / 2)$ which reduces to the result given by Gold, ${ }^{9} G_{\text {STLS }}(\gamma \rightarrow 0) \approx(2 / \pi) \gamma^{1 / 2}$, as $a \rightarrow 0$. In the Vashishta-Singwi theory ${ }^{17}$ the parameter $a$ is determined by adjusting the compressibility calculated using the groundstate energy and that obtained from the long-wavelength limit of the dielectric function. In this work we take $a=1 / 2$ which gives the best agreement with the exact ground state energy. We discuss the compressibility sum rule in the subsequent sections. 


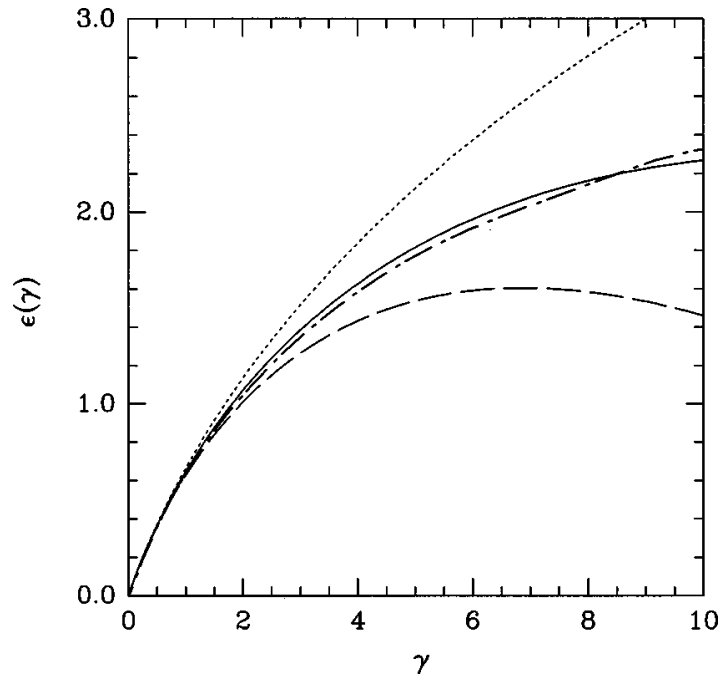

FIG. 2. The ground-state energy per particle $\epsilon(\gamma)$, in units of $n^{2} / 2 m$, as a function of the coupling strength $\gamma$. The dotted and dot-dashed lines are for the STLS approximation (Ref. 9) and the exact result of Ref. 5, respectively. The solid and dashed lines represent the VS approximation for $a=1 / 2$ and $a=1$, respectively.

\section{GROUND STATE ENERGY}

The interaction energy (per particle) of a many particle system is written as $E_{\text {int }}(\gamma)=(1 / 2) \Sigma_{q} V_{q}\left[N \delta_{q=0}+S(q)-1\right]$, in which the Hartree contribution is also included. Within the mean-field theory this reduces to $E_{\text {int }}(\gamma)=\left(n^{2} / 2 m\right)[1$ $\left.-2 \gamma^{1 / 2}(1-G(\gamma))^{1 / 2} / \pi\right]$. The ground state energy per particle is calculated by a coupling constant integration $E_{0}$ $=\int_{0}^{\gamma} d \lambda E_{\mathrm{int}}(\lambda) / \lambda$. We express $E_{0}$ in terms of the dimensionless quantity given by

$$
\epsilon(\gamma)=\int_{0}^{\gamma} d \lambda\left[1-\frac{2}{\pi} \lambda^{1 / 2}\left(1-G_{\mathrm{VS}}(\lambda)\right)^{1 / 2}\right],
$$

where $E_{0}=\left(n^{2} / 2 m\right) \epsilon(\gamma)$.

In the exact treatment of $\epsilon(\gamma)$, an expansion for small $\gamma$ was not provided because of the inadequacy of the perturbation theory and nonanalytic properties of $\epsilon(\gamma)$ as $\gamma \rightarrow 0$. Gold $^{9}$ has shown that the local-field correction becomes a useful quantity in the analysis of $1 \mathrm{D}$ bosons and has given a weak coupling expansion

$$
\begin{gathered}
\epsilon_{\text {STLS }}(\gamma \rightarrow 0)=\gamma-\frac{4}{3 \pi} \gamma^{3 / 2}+\frac{1}{\pi^{2}} \gamma^{2}-\frac{2}{5 \pi^{3}} \gamma^{5 / 2}+\frac{1}{14 \pi^{5}} \gamma^{7 / 2} \\
+\cdots
\end{gathered}
$$

As may be seen in Fig. 2, the STLS approximation compares well with the exact result of Lieb and Liniger ${ }^{5}$ only for $\gamma$ $\lesssim 2$. Our Vashishta-Singwi approach yields the following weak coupling expansion (for $a=1 / 2$ )

$$
\begin{gathered}
\epsilon_{\mathrm{VS}}(\gamma \rightarrow 0)=\gamma-\frac{4}{3 \pi} \gamma^{3 / 2}+\frac{3}{4 \pi^{2}} \gamma^{2}-\frac{7}{40 \pi^{3}} \gamma^{5 / 2}-\frac{5}{192 \pi^{4}} \gamma^{3} \\
-\frac{11}{3584 \pi^{5}} \gamma^{7 / 2}+\cdots
\end{gathered}
$$

Figure 2 shows that the VS approach adopted here gives a better agreement than the STLS result to the exact ground-

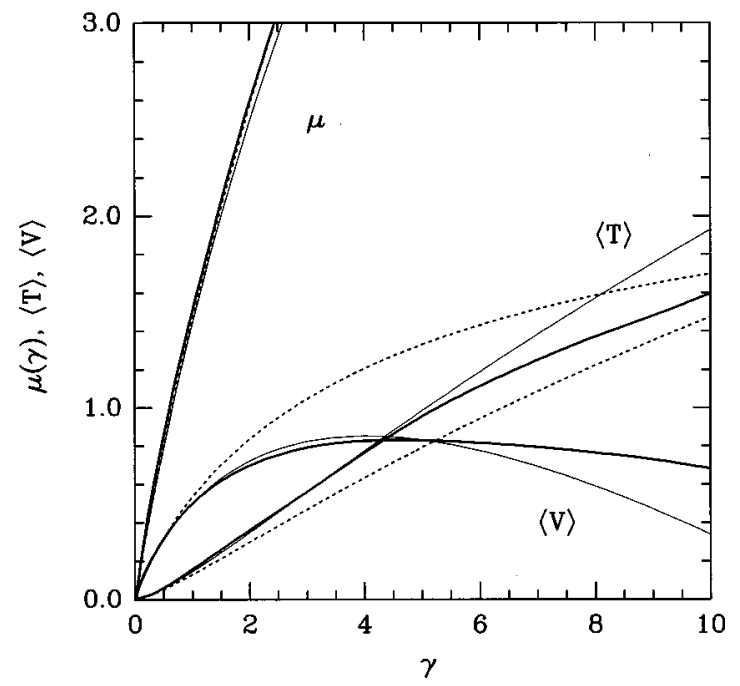

FIG. 3. The chemical potential, average kinetic and potential energies as functions of $\gamma$. The thick solid lines denote the exact results of Ref. 5. The thin solid lines and dotted lines are for the VS and STLS approximations (Ref. 9), respectively.

state energy $\epsilon(\gamma)$. More interestingly, the comparison of weak coupling expansions of $\epsilon_{\mathrm{STLS}}(\gamma)$ and $\epsilon_{\mathrm{VS}}(\gamma)$ demonstrates that terms of order $\gamma^{n}$ where $n \geqslant 3$ are missing in the $\epsilon_{\text {STLS }}(\gamma)$ expansion. Some other ground state quantities of interest are the chemical potential

$$
\mu=\partial E_{0} / \partial N=\left(n^{2} / 2 m\right)(3 \epsilon-\gamma d \epsilon / d \gamma),
$$

the average potential energy per particle $\langle V\rangle$ $=\left(n^{2} / 2 m\right) \gamma d \epsilon / d \gamma$, and the average kinetic energy $\langle T\rangle=E_{0}$ $-\langle V\rangle=\left(n^{2} / 2 m\right)(\epsilon-\gamma d \epsilon / d \gamma)$. Using the numerically calculated $\epsilon_{\mathrm{VS}}(\gamma)$ we compare these quantities with the results of the exact solution to the 1D boson problem in Fig. 3. We first note that the STLS approximation results (dotted lines) start deviating from the exact calculation of $\langle T\rangle$ and $\langle V\rangle$ for $\gamma$ $\approx 2$. The VS calculation represents $\langle T\rangle$ reasonably well, but the potential energy term starts to deviate from the exact result for $\gamma \geq 6$. However a cancelation effect renders the total energy in quantitative agreement with the exact result up to $\gamma \approx 10$ (see Fig. 2). In the available range of $\gamma$ both the STLS and VS approximations agree well with the exact result for the chemical potential $\mu(\gamma)$. The weak coupling limits of the chemical potential and the average potential and kinetic energies per particle (in units of $n^{2} / 2 m$ ) in the VS approximation are given by

$$
\mu=2 \gamma-\frac{2}{\pi} \gamma^{3 / 2}+\frac{3}{4 \pi^{2}} \gamma^{2}-\frac{7}{80 \pi^{3}} \gamma^{5 / 2}+\frac{11}{7168 \pi^{5}} \gamma^{7 / 2}+\cdots,
$$

$$
\begin{aligned}
\langle V\rangle= & \gamma-\frac{2}{\pi} \gamma^{3 / 2}+\frac{3}{2 \pi^{2}} \gamma^{2}-\frac{7}{16 \pi^{3}} \gamma^{5 / 2}-\frac{5}{64 \pi^{4}} \gamma^{3} \\
& -\frac{11}{1024 \pi^{5}} \gamma^{7 / 2}+\cdots,
\end{aligned}
$$




$$
\begin{aligned}
\langle T\rangle= & \frac{2}{3 \pi} \gamma^{3 / 2}-\frac{3}{4 \pi^{2}} \gamma^{2}+\frac{21}{80 \pi^{3}} \gamma^{5 / 2}+\frac{5}{96 \pi^{4}} \gamma^{3} \\
& +\frac{55}{7168 \pi^{5}} \gamma^{7 / 2}+\cdots .
\end{aligned}
$$

When we perform a similar calculation using the groundstate energy within the STLS approximation, ${ }^{8,9}$ we obtain

$$
\begin{aligned}
& \mu=2 \gamma-\frac{2}{\pi} \gamma^{3 / 2}+\frac{1}{\pi^{2}} \gamma^{2}-\frac{1}{5 \pi^{3}} \gamma^{5 / 2}-\frac{1}{28 \pi^{5}} \gamma^{7 / 2}+\cdots, \\
& \langle V\rangle=\gamma-\frac{2}{\pi} \gamma^{3 / 2}+\frac{2}{\pi^{2}} \gamma^{2}-\frac{1}{\pi^{3}} \gamma^{5 / 2}+\frac{1}{4 \pi^{5}} \gamma^{7 / 2}+\cdots \\
& \langle T\rangle=\frac{2}{3 \pi} \gamma^{3 / 2}-\frac{1}{\pi^{2}} \gamma^{2}+\frac{3}{5 \pi^{3}} \gamma^{5 / 2}-\frac{5}{28 \pi^{5}} \gamma^{7 / 2}+\cdots
\end{aligned}
$$

which again shows that certain terms are missing in the expansion of $\langle V\rangle$ and $\langle T\rangle$ compared to the VS approximation.

\section{COLLECTIVE EXCITATIONS}

The collective mode excitation spectrum in our model is readily obtained from the RPA-like density-density response function. The dispersion relation for the collective mode is given by $^{9}$

$$
\omega_{q}=\left(\frac{n^{2}}{2 m}\right) \frac{q}{n}\left[(q / n)^{2}+4 \gamma(1-G(\gamma))\right]^{1 / 2},
$$

which represents a gapless excitation (may be identified as the Goldstone mode). Taking $G(\gamma)=0$ or its weak coupling limit $G(\gamma) \approx 2 \gamma^{1 / 2} / \pi$ yields the collective mode dispersion in the RPA and the Bogoliubov approximation, respectively. In the exact solution ${ }^{6}$ of the interacting $1 \mathrm{D}$ boson gas two types of elementary excitations were found, the first of which ("particle" excitations) corresponds to the Bogoliubov spectrum. The second type ("hole"' excitations) of the elementary excitation which exists only for $|q|<\pi n$, is not accounted for within the Bogoliubov approximation or the present model with a local-field correction. Part of the reason for this is that the Bogoliubov model assumes that all the particles are in the condensate whereas in the treatment of Lieb and Liniger ${ }^{5,6}$ no such assumption is made. In the recent work of Kerman and Tommasini ${ }^{12}$ the self-interactions of the particles out of the condensate are taken into account. Although the Gaussian variational method provides only an upper bound for the ground state energy and does not reproduce the exact energy so well for $\gamma \gtrsim 5$, it captures successfully the basic structure of the elementary excitations.

\section{SOUND VELOCITY}

The VS theory was originally devised ${ }^{17}$ to fulfill the compressibility sum rule in interacting electron systems. It was demonstrated that the compressibility calculated from the long-wavelength limit of the response function coincides with that calculated from the ground-state energy through the

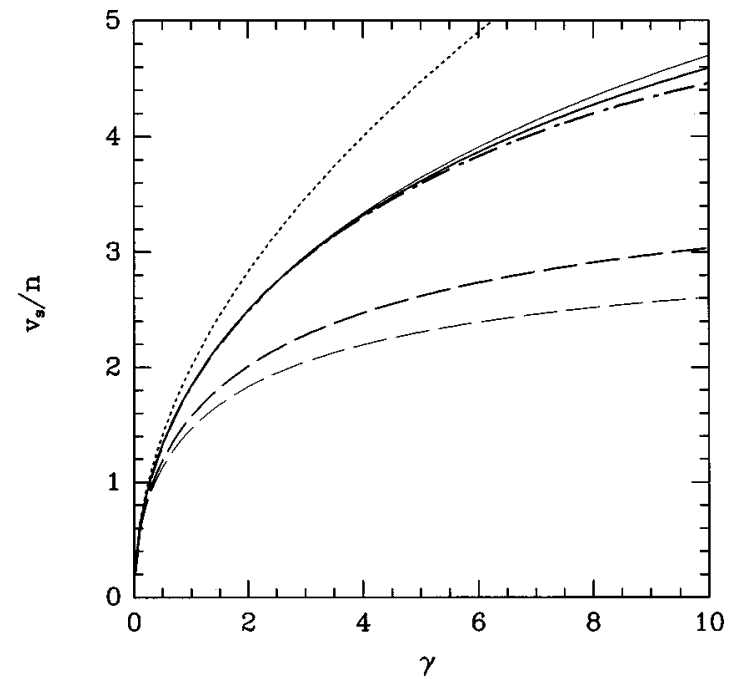

FIG. 4. The velocity of sound $v_{s}$ as a function of $\gamma$. The lower (dashed) and upper (solid) curves are calculated from the excitation spectrum and thermodynamic definition, respectively. The thick and thin lines are for the VS and STLS approximations (Ref. 9), respectively. The exact result from Ref. 6 is depicted by the dot-dashed line. $v_{s}$ calculated using the Bogoliubov excitation spectrum is given by the dotted line.

thermodynamic relation. Since the compressibility is also related to the velocity of sound by $1 / \kappa=m n v_{s}^{2}$, we can use the sound velocity in the present context to check the compressibility sum rule. The sound velocity may be calculated from the excitation spectrum $v_{s}=\lim _{q \rightarrow 0} \partial \omega_{q} / \partial q$ which yields the result $v_{s}=2 n[4 \gamma(1-G(\gamma))]^{1 / 2}$. On the other hand, the thermodynamic relation $v_{s}^{2}=\left(\gamma^{2} / m\right) \partial^{2} E_{0} / \partial \gamma^{2}$, gives

$$
v_{s}=2\left[\gamma^{2} \frac{\frac{1}{2} \partial^{2} \epsilon}{\partial \gamma^{2}}-2 \gamma \frac{\partial \epsilon}{\partial \gamma}+3 \epsilon\right]^{1 / 2} .
$$

In Fig. 4 we show the velocity of sound calculated in the VS and STLS approximations using the above mentioned two different ways. The ground-state energy based calculation of $v_{s}$ within the STLS and VS approximations are quite close to the exact result for $\gamma \lesssim 10$. The excitation energy spectrum based calculation of $v_{s}$ remains below the thermodynamic results. In the VS approach the compressibility sum rule is violated less than in the STLS approach, but it is still not very satisfactory. The Bogoliubov spectrum yields a sound velocity above the energy based results. It is not surprising that the compressibility sum rule is not satisfied either in the STLS or the VS approximations, since both of these schemes assume that all the particles are in the condensate. In other words, the local-field based theories take only the "particle" contribution to the excitation spectrum and neglect the "hole" excitations.

\section{STATIC STRUCTURE FACTOR AND PAIR-CORRELATION FUNCTION}

The static structure factor as defined in Eq. (2) gives a measure of the correlation effects. For a noninteracting system it is unity and in the random-phase approximation (RPA) 


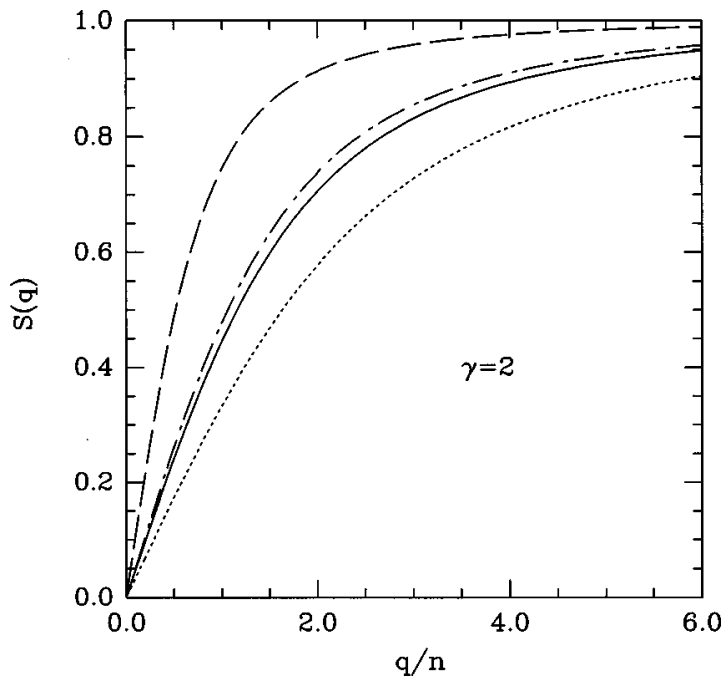

FIG. 5. The static structure factor $S(q)$ at $\gamma=2$ for various approximations. The solid and dot-dashed lines indicate $S_{\mathrm{VS}}(q)$ and $S_{\text {STLS }}(q)$ (Ref. 9), respectively. The dotted and dashed lines are for the RPA and Bogoliubov approximations, respectively.

we take $G(\gamma)=0$. The Bogoliubov approximation is recovered when the leading order term in the local-field factor is retained, viz. $G(\gamma)=2 \gamma^{1 / 2} / \pi$. Figure 5 shows the static structure factor $S(q)$ for $\gamma=2$ in the RPA, Bogoliubov, STLS and the present VS approximations. In the RPA the interaction effects are over-stressed. The Bogoliubov approximation is closest to the noninteracting result, but for $\gamma$ $=2$ it may already be not so good. The inclusion of the local-field correction $G(\gamma)$ tends to decrease $S(q)$ from the Bogoliubov result, since in the latter correlation effects are not fully taken into account. The probability of finding two bosons at a distance $r$ is described by the pair-correlation function $g(r)$ which is the Fourier transform of $S(q)$. Performing the one-dimensional Fourier integral analytically ${ }^{20}$ we obtain the pair-correlation function within the present model as

$$
\begin{aligned}
g_{\mathrm{VS}}(r)= & g_{\mathrm{VS}}(0)+\gamma^{1 / 2}\left(1-G_{\mathrm{VS}}\right)^{1 / 2}\left[I_{1}\left(2 r n \gamma^{1 / 2}\left(1-G_{\mathrm{VS}}\right)^{1 / 2}\right)\right. \\
& \left.-\mathbf{L}_{1}\left(2 r n \gamma^{1 / 2}\left(1-G_{\mathrm{VS}}\right)^{1 / 2}\right)\right],
\end{aligned}
$$

where $I_{1}(x)$ is the modified Bessel function (of the first kind), $\mathbf{L}_{1}(x)$ is the modified Struve function, ${ }^{20}$ and $g_{\mathrm{vs}}(0)$ $=1-(2 / \pi) \gamma^{1 / 2}\left(1-G_{\mathrm{VS}}\right)^{1 / 2}$ is the pair-correlation function at zero separation. Note that a similar expression for the pair-correlation function within the STLS was also given by Gold. ${ }^{9}$ Figure 6 compares $g_{\text {vS }}(0)$ with $g_{\text {STLS }}(0)$ as a function of $\gamma$. It was noted that in the STLS approximation $g(0)$ remains positive for all $\gamma$, unlike the Coulomb systems which yield unphysically negative $g(0)$ at some intermediate coupling strength. In the case of Vashishta-Singwi approximation, we find that $g(0)$ eventually becomes negative for $\gamma \gtrsim 15$. Since the theories involving the local-field factor are perturbational in character, thus limiting their applicability for small and intermediate range of $\gamma$, our result for $g_{\mathrm{vS}}(0)$ should be useful in practical applications. The weak coupling limit of $g_{\mathrm{VS}}(0)$ is given by

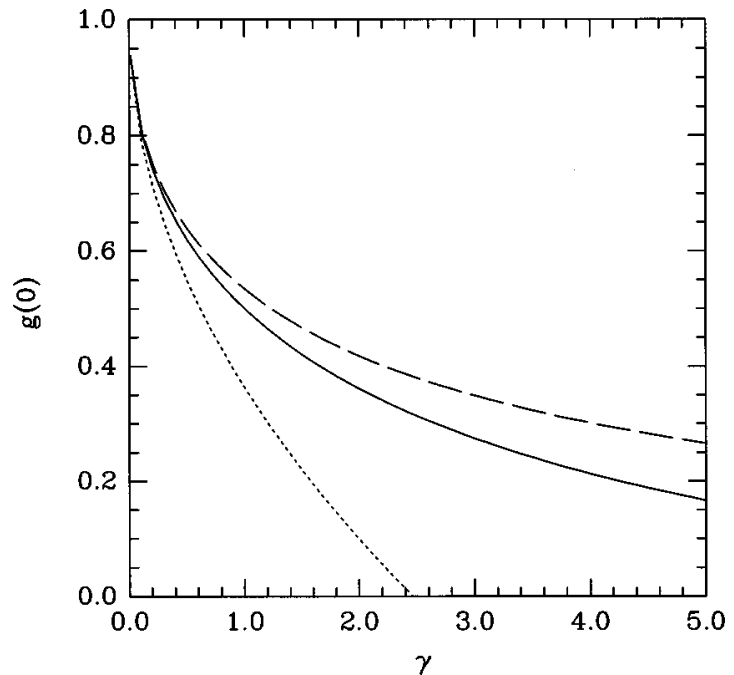

FIG. 6. The pair-correlation function at zero separation $g(0)$ as a function of $\gamma$. The solid, dashed, and dotted lines indicate $g_{\mathrm{VS}}(0)$, $g_{\mathrm{STLS}}(0)$ (Ref. 9), and $g(0)$ in the Bogoliubov approximation, respectively.

$$
\lim _{\gamma \rightarrow 0} g_{\mathrm{VS}}(0) \simeq 1-\frac{2}{\pi} \gamma^{1 / 2}+\frac{2}{\pi^{2}} \gamma(1-a / 2)+\cdots,
$$

which reduces to the STLS result ${ }^{9} g_{\text {STLS }}(0) \simeq 1-2 \gamma^{1 / 2} / \pi$, as $a \rightarrow 0$. The effect of the STLS and VS local-field corrections on the pair-correlation function is further illustrated in Fig. 7 where $g(r)$ is plotted for $\gamma=1$ and $\gamma=5$, and we also specialize to the $a=1 / 2$ case. The differences occur largely at small separations. The asymptotic forms of $g(r)$ are obtained as

$$
\begin{aligned}
g_{\mathrm{VS}}(r \rightarrow 0)= & g_{\mathrm{VS}}(0)+r n \gamma\left[1-G_{\mathrm{VS}}(\gamma)\right] \\
& -\frac{8 r^{2} n^{2}}{3 \pi} \gamma^{3 / 2}\left[1-G_{\mathrm{VS}}(\gamma)\right]^{3 / 2}+\cdots
\end{aligned}
$$

and

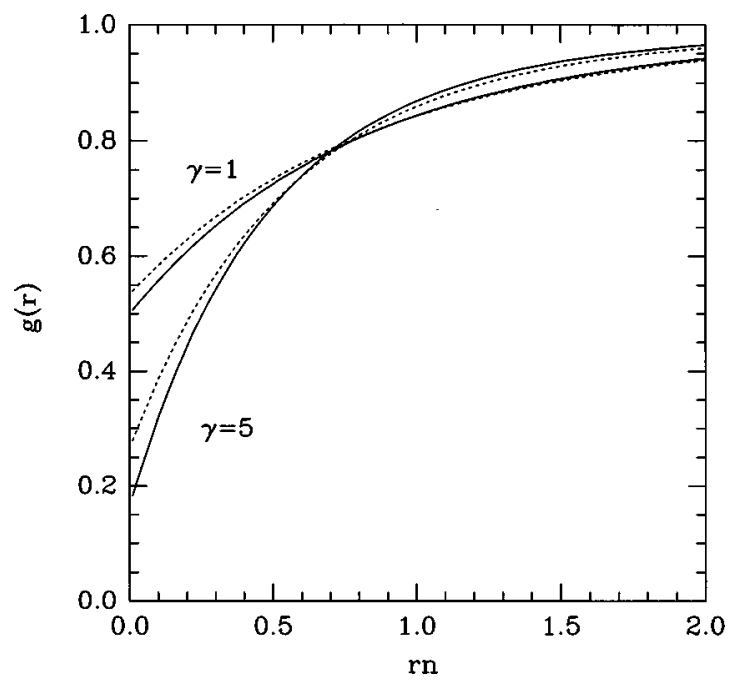

FIG. 7. The pair-correlation function $g(r)$ at $\gamma=1$ and $\gamma=5$ as a function of $r$. The solid and dotted lines indicate $g_{\mathrm{VS}}(r)$ and $g_{\mathrm{STLS}}(r)$ (Ref. 9), respectively. 


$$
\begin{aligned}
g_{\mathrm{VS}}(r \rightarrow \infty) & =1-\frac{2}{\pi r^{2} n^{2}} \gamma^{-1 / 2}\left[1-G_{\mathrm{VS}}(\gamma)\right]^{-1 / 2} \\
& -\frac{3}{2 \pi r^{4} n^{4}} \gamma^{-3 / 2}\left[1-G_{\mathrm{VS}}(\gamma)\right]^{-3 / 2}+\cdots
\end{aligned}
$$

A comparison with the corresponding expansions in the STLS approximation ${ }^{9}$ shows that both methods have the same $r$ dependences.

\section{DISCUSSION}

In this work we have employed the formalism of Vashishta and Singwi ${ }^{17}$ to study the ground state properties of a system of 1D boson condensates. The VS approach is largely based on the STLS theory. The local-field factor which successfully describes the correlation effects in electron liquids is found also to be useful in the present analysis. In comparison to the STLS approach, the VS local-field description extends the validity range of approximate theories to $\gamma \lesssim 10$, since for this region the ground-state energy is faithfully reproduced. The strong coupling regime, viz. $\gamma \rightarrow \infty$ is not described well by the STLS and VS approaches, thus we have omitted any discussion on this regime. In the dielectric formulation of the interacting quantum systems the sum-rule constraints $^{21}$ on the frequency and wave vector dependent dielectric function $\varepsilon(q, \omega)$ is often found useful. We point out that the frequency and wave vector independent localfield corrections satisfy the first and third moment sum-rules simultaneously. This follows from the fact that the local-field factor is independent of wave vector variable $q$. However, it is conceivable to imagine the correlation effects giving rise to an effective $q$-dependent interaction given by $V_{0}[1$ $-G(q ; \gamma)]$, even though the bare interaction $V_{0}$ is a constant. This may be achieved within the so-called SSTL approach of Singwi et al. ${ }^{22}$ in which the bare Coulomb interaction is renormalized by the static screening function $\varepsilon(q)$ which is incorporated into the self-consistent scheme. The consequences of the SSTL approach with wave vector dependent local-field correction in the context of 1D bosons appears to be an interesting problem for future explorations.

The major shortcoming of the present model (as well as the Bogoliubov and STLS approaches) is that the 1D bosons are assumed to be in the condensate. It is known that the 1D Bose system at zero temperature cannot have long range order and the Bose condensation in this system is absent. ${ }^{19,23}$ In fact, our effort to calculate the depletion of the condensate as a result of particle interactions was fruitless. The inequality $n_{q} \geqslant n_{0} / 4 S(q)-1 / 2$, for the momentum distribution which must be obeyed for Bose systems satisfying the $f$-sum rule, ${ }^{23}$ yields negative values for $q / n \gtrsim 2$. However, it is interesting to observe that the assumption of full condensation yields ground state energy and other properties in reasonable agreement with the exact results. Combining our results and recalling the earlier results of Gold ${ }^{9}$ and those of Kerman and Tommasini $^{12}$ we conclude that the condensate assumption is not very severe for the ground state energy, but the interaction with out of condensate particles is essential for the description of elementary excitations. Because of the recent interest in Bose-Einstein condensed gases in lowdimensional systems, we believe our results should be useful in understanding the interaction effects on the condensed systems. One possible way to improve upon the condensate assumption is to consider a two fluid model, and take the momentum distribution of the out of the condensate particles explicitly into account. Further work is necessary to explore these ideas.

Both the STLS and VS approximations are based on the perturbation theory, thus they are not expected to yield reliable results in the strong coupling limit, $\gamma \rightarrow \infty$. In particular, $G_{\mathrm{VS}}(\gamma)$ behaves as $\sim 1-\pi^{2}(1+a) / 4 \gamma$ for large $\gamma$. A possible improvement may be achieved if one considers the ladder diagrams (multiple scattering) which take the short-range correlations into account in an improved way. Such an approach was shown to work quite well for 1D fermions interacting with a $\delta$ function. ${ }^{24}$

In summary, we have considered the correlation effects in a system of $1 \mathrm{D}$ bosons interaction with a short-range potential. We have introduced a local-field factor based on the Vashishta-Singwi approach to describe the correlation effects. The ground state energy calculated within this approximation is in good agreement with the exacts results for $\gamma$ $\lesssim 10$, thus showing an improvement compared to the Bogoliubov and STLS approximations. The sound velocity and correlation functions in the present model are also calculated.

\section{ACKNOWLEDGMENTS}

This work was partially supported by the Scientific and Technical Research Council of Turkey (TUBITAK) under Grant No. TBAG-1662. We thank Dr. C. Bulutay for fruitful discussions, and Professor M. Gürses and Dr. U. Muğan for valuable comments.
${ }^{1}$ The Many-body Problem, edited by D. C. Mattis (World Scientific, Singapore, 1993).

${ }^{2}$ P. Nozières and D. Pines, The Theory of Quantum Liquids Volume II: Superfluid Bose Liquids (Addison-Wesley, Redwood City, CA, 1990).

${ }^{3}$ C. N. Yang, Phys. Rev. Lett. 19, 1312 (1967).

${ }^{4}$ M. Girardeau, J. Math. Phys. 1, 516 (1960).

${ }^{5}$ E. H. Lieb and W. Liniger, Phys. Rev. 130, 1605 (1963).

${ }^{6}$ E. H. Lieb, Phys. Rev. 130, 1616 (1963).

${ }^{7}$ C. N. Yang and C. P. Yang, J. Math. Phys. 10, 1115 (1969).
${ }^{8}$ O. Hipôlito and R. Lobo, Phys. Rev. B 17, 1433 (1978).

${ }^{9}$ A. Gold, Z. Phys. B 91, 397 (1993).

${ }^{10}$ K. S. Singwi, M. P. Tosi, R. H. Land, and A. Sjölander, Phys. Rev. 176, 589 (1968).

${ }^{11}$ N. N. Bogoliubov, J. Phys. (Moscow) 11, 23 (1947).

${ }^{12}$ A. K. Kerman and P. Tommasini, Phys. Rev. B 56, 14733 (1997).

${ }^{13}$ T. Blum, D. S. Koltun, and Y. Shapir, Phys. Rev. Lett. 66, 2417 (1991).

${ }^{14}$ A. Gold and L. Calmels, Phys. Rev. B 57, 12119 (1998). 
${ }^{15}$ E. H. Lieb and J. Yngvason, Phys. Rev. Lett. 80, 2504 (1998).

${ }^{16}$ M. H. Anderson, J. R. Ensher, M. R. Matthews, C. E. Wieman, and E. A. Cornell, Science 269, 198 (1995); K. B. Davis, M.-O. Mewes, M. R. Andrews, N. J. van Druten, D. S. Durfee, D. M. Kurn, and W. Ketterle, Phys. Rev. Lett. 75, 3969 (1995); M.-O. Mewes, M. R. Andrews, N. J. van Druten, D. M. Kurn, D. S. Durfee, and W. Ketterle, ibid. 77, 416 (1996).

${ }^{17}$ P. Vashishta and K. S. Singwi, Phys. Rev. B 6, 875 (1972).

${ }^{18}$ S. Conti, M. L. Chiofalo, and M. P. Tosi, J. Phys.: Condens. Matter 6, 8795 (1994).
${ }^{19}$ P. C. Hohenberg, Phys. Rev. 158, 383 (1967); N. D. Mermin, ibid. 176, 250 (1968).

${ }^{20}$ I. S. Gradshteyn and I. M. Rhyzik, Table of Integrals, Series and Products (Academic Press, New York, 1980).

${ }^{21}$ N. Iwamoto, Phys. Rev. A 30, 3289 (1984).

${ }^{22}$ K. S. Singwi, A. Sjölander, M. P. Tosi, and R. H. Land, Phys. Rev. B 1, 1044 (1970).

${ }^{23}$ L. Pitaevskii and S. Stringari, J. Low Temp. Phys. 85, 377 (1991).

${ }^{24}$ S. Nagano and K. S. Singwi, Phys. Rev. B 27, 6732 (1983). 